\title{
FASE SENSORA DE POLI(CLORETO DE VINILA) NA DETECÇÃO DE 17ß-ESTRADIOL EM MEIO AQUOSO UTILIZANDO ESPECTROSCOPIA NO INFRAVERMELHO MÉDIO E QUIMIOMETRIA
}

\author{
Tamires da Silva Limaa, Gabriel Bercley de Lima Vitorinoa, Elaine Cristina Lima do Nascimentoa Carlos André de Souzaa,

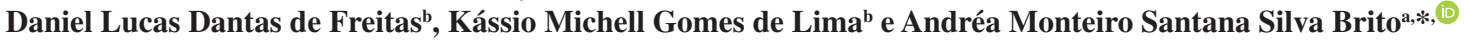 \\ aUnidade Acadêmica de Serra Talhada, Universidade Federal Rural de Pernambuco, 56909-535 Serra Talhada - PE, Brasil \\ 'Instituto de Química, Química Biológica e Quimiometria, Universidade Federal do Rio Grande do Norte, 59072-970 Natal - RN, \\ Brasil
}

Recebido em 10/03/2020; aceito em 09/06/2020; publicado na web em 15/07/2020

\begin{abstract}
SENSING PHASE OF POLY(VINYL CHLORIDE) IN THE DETECTION OF 17 $\beta$-ESTRADIOL IN WATER ENVIRONMENT USING MID-INFRARED SPECTROSCOPY AND CHEMOMETRICS. In recent years, estrogens have gained notable attention among emerging pollutants, increasingly leading to the need for less laborious analytical methods. Optical sensors are a promising technology for the determination of chemical contaminants in water, acting as an alternative screening method. The aim of this work was to evaluate the performance of poly(vinyl chloride) (PVC) as a sensing phase for the detection of $17 \beta$-estradiol in water using mid-infrared spectroscopy and chemometrics. The sensing phase was used for differentiation purposes with an accuracy of $80 \%$, sensitivity of $100 \%$ and specificity of $71.4 \%$ based on soft independent modeling of class analogy (SIMCA). Satisfactory limits of detection $\left(8.9 \times 10^{-1}-2.9 \mathrm{mg} \mathrm{L}^{-1}\right)$, quantification $\left(2.9-1.0 \times 10 \mathrm{mg} \mathrm{L}^{-1}\right)$, slope of the analytical curve $\left(5.0 \times 10^{-4}-2.4 \times 10^{-3} \mathrm{~L} \mathrm{mg}^{-1}\right)$ and determination coefficients $(0.90-0.99)$ were obtained. Kinetically, a extraction time of around 30 min was estimated and in a regeneration study, it was possible to use the same phase after 4 cycles of use. Therefore, the results indicate that the PVC sensing phase is promising for the detection of $17 \beta$-estradiol in water by means of mid-infrared spectroscopy and chemometric methods.
\end{abstract}

Keywords: water; $17 \beta$-estradiol; PVC; infrared spectroscopy; SIMCA.

\section{INTRODUÇÃO}

Do extenso rol de compostos considerados contaminantes emergentes, encontram-se os fármacos, hormônios, alquifenóis e seus derivados, drogas ilícitas, pesticidas, compostos perfluorados, percloratos, microplásticos. ${ }^{1-8}$

A literatura reporta que estes compostos podem ser classificados como interferentes endócrinos por seu potencial ou capacidade de alterarem as funções do sistema endócrino de humanos e de outros animais, afetando a saúde, o crescimento e a reprodução, podendo causar efeitos adversos em organismos saudáveis ou em seus descendentes. ${ }^{9}$

Nos últimos anos, uma diversidade de hormônios naturais e sintéticos compreendidos como interferentes endócrinos tem despertado a atenção de comunidade científica. O 17ß-estradiol (E2), por exemplo, é um estrogênio natural essencial para o desenvolvimento e manutenção de tecidos reprodutivos femininos, que se apresenta como um contaminante de risco, pois estudos sinalizam que na faixa de concentrações em ng L $\mathrm{L}^{-1}$ esse contaminante pode ocasionar problemas acentuados de fertilidade das mulheres, aumentando o risco de exposição ao câncer de ovário e de mama e também têm provocado a mutação de várias espécies de peixes, oriundos da exposição crônica a esse hormônio. ${ }^{8-10}$

No entanto, vale ressaltar, que para os contaminantes emergentes ainda não existe uma legislação indicando níveis permitidos no ambiente. Alguns pesquisadores já sinalizam que uma futura regulamentação depende de resultados obtidos em estudos de ecotoxicidade, efeitos à saúde humana, potencial de bioacumulação, transporte e destino nos diferentes compartimentos ambientais, além da quantidade em que são lançados, ou seja, a concentração no ambiente. ${ }^{6}$

*e-mail: andrea.monteiros@ufrpe.br
Geralmente, a detecção desses contaminantes em meio aquoso é realizada por métodos cromatográficos, dentre eles a cromatografia gasosa (GC), a cromatografia líquida de alta eficiência (HPLC), a cromatografia gasosa com extração headspace múltipla (MHE-GC) e cromatografia líquida acoplada à espectrometria de massas sequencial (LC-MS/MS). ${ }^{6,11-13}$

Apesar de sensíveis, o custo dos experimentos envolvendo esses métodos é relativamente elevado. Além disso, nas técnicas cromatográficas, em geral, os procedimentos são laboriosos, apresentam dificuldades para monitoramento em campo e necessitam de solventes, reagentes e soluções padrões que podem gerar resíduos prejudiciais ao ambiente. ${ }^{14-19}$

Como alternativa para auxiliar na detecção de hormônios em meio aquoso, alguns métodos são investigados. No trabalho de Raymundo-Pereira e colaboradores ${ }^{14}$ é reportado o uso da detecção eletroquímica, com dados de voltametria de pulso diferencial para detectar o hormônio estriol em meio aquoso, com limites de detecção e quantificação de 0,04 e $0,16 \mathrm{mg} \mathrm{L}^{-1}$, respectivamente. $\mathrm{O}$ trabalho mostrou-se particularmente importante para o futuro desenvolvimento de ferramentas analíticas miniaturizadas com baixo custo e procedimentos simples de fabricação. Métodos relacionando sensores à espectroscopia no infravermelho também têm sido desenvolvidos para determinação de compostos orgânicos em água. ${ }^{15-17,19-28}$ Muitos autores têm indicado por vários anos a robustez do uso da detecção espectroscópica, sendo possível estender a aplicação para uma ampla gama de situações contínuas de monitoramento de poluição, monitoramento precoce, monitoramento de efluentes e análise de água potável. ${ }^{18,28-31}$

Em 2016, os pesquisadores Hass e Mizaikoff ${ }^{18}$ descreveram os avanços da espectroscopia de infravermelho médio (MID) para análise química. Nesse trabalho, os autores mostraram como evoluiu esta faixa de detecção no infravermelho, passando de simples técnica de laboratório de rotina para a espectroscopia de ponta, se tornando uma 
ferramenta de sensoriamento, de aquisição de espectros sofisticados, tendo aumentado a sensibilidade, a velocidade de aquisição de dados e a resolução espectral. Atendendo as aplicações exigentes e inovadoras, como por exemplo, em análise de processos da biotecnologia a diagnóstico médico. Assim, espera-se que a interface das técnicas analíticas existentes se expanda no espaço de informações/dados, enquanto diminui os custos de análise associadas, trabalhando no sentido de avaliação precisa, de forma mais rápida e com menor custo.

Em particular, os métodos analíticos, que utilizam a espectroscopia na região do infravermelho, desenvolvidos para análise em meio aquoso, baseiam-se em estratégias que eliminam a água da medida, geralmente, os poluentes estão presentes na água em quantidade pequena e a determinação direta é difícil. ${ }^{30}$ Assim, se os analitos presentes na água forem transferidos e concentrados numa fase sólida (um polímero, por exemplo), haverá a possibilidade de determinação. Esse artifício é conhecido como microextração em fase sólida (SPME) na cromatografia, que consiste em concentrar o contaminante ambiental para posterior detecção. Na região do infravermelho, a SPME é adaptada e utilizada por meio de técnicas de reflexão total atenuada (ATR), transflectância e transmitância, sendo esta fase sólida chamada de fase sensora. ${ }^{15-19}$

Dentro dessa abordagem, uma aplicação relevante nessa área é a dos sensores com detecção no infravermelho que podem atuar futuramente como métodos de rastreamento, reconhecimento e varredura, conhecidos como screening. O objetivo desse método é obter dados analíticos para posterior definição de uma caracterização mais detalhada do local afetado e também orientar futuros planos de monitoramento e recuperação. Dentre as possibilidades analíticas de screening, os sensores químicos ópticos são classificados como uma tecnologia que pode oferecer muito na identificação e quantificação de compostos. . $^{15,17-19,30,31}$

Os sensores químicos ópticos fornecem informações sobre a presença de compostos específicos em amostras. Para tanto, eles precisam de um componente principal imobilizado (fase sensora), através do qual ocorre a geração do sinal óptico proporcional à concentração do analito a ser determinado. ${ }^{16-19}$

A utilização destas fases sensoras tem como finalidade reduzir interferências espectrais que são referentes às bandas largas de $\mathrm{O}-\mathrm{H}$ da água nos espectros de infravermelho, pois excluem a água das medidas, como bem justificado no trabalho de Silva e colaboradores. ${ }^{30}$ Essas bandas frequentemente dificultam ou até mesmo inviabilizam a determinação dos compostos orgânicos em meio aquoso utilizando diretamente a técnica de espectroscopia de infravermelho. ${ }^{15-19,30}$

Os métodos envolvendo sensores e a espectroscopia no infravermelho, a partir de fases sensoras, apresentam vantagens por serem de baixo custo, simples e podem ser seletivas, possibilitando a obtenção de resultados confiáveis. ${ }^{17-18,30}$ Frequentemente são publicados artigos que focam na investigação de polímeros que possam atuar como meio de pré-concentração de contaminantes orgânicos em águas. ${ }^{16-23,27-30}$

Dentre os polímeros reportados na literatura, destacam-se o poli(cloreto de vinila) (PVC), polidimetilsiloxano (PDMS), polisobutileno (PIB) e poliuretano (PU). ${ }^{16-17,19,30-36}$ Nesses trabalhos, os materiais poliméricos apresentaram boas interações com contaminantes orgânicos e limites de detecção em baixas concentrações.

No presente trabalho foi realizado um estudo com a matriz polimérica de PVC. Nossa escolha por esse polímero se deu pelo fato de ainda não ter sido reportada na literatura uma fase sensora de PVC aplicado na determinação de hormônios em água. Além disso, existe um baixo custo associado aos filmes poliméricos de PVC, bem como estes filmes são de fácil preparação e não interagem com a água, características importantes para compor as fases sensoras para o sistema óptico. Assim, o trabalho desenvolvido visou contribuir cientificamente para o fortalecimento do conhecimento na área, antecipando uma possibilidade de material polimérico para uso como fase sensora em sistema com detecção óptica, ou em outros métodos que precisem selecionar uma fase sólida que extraia o E2 do ambiente aquoso.

Como ferramentas para o desenvolvimento do método do presente estudo foi empregada a técnica de reflexão total atenuada (UATR) no infravermelho médio com transformada de Fourier (FTIR) associada a métodos de reconhecimento de padrões, sendo eles o método de análise exploratória PCA (Principal Component Analysis) e o método de classificação SIMCA (Soft Independent Modeling by Class Analogy).

Esses métodos de reconhecimento de padrões visam classificar dados baseados em conhecimento a priori (preliminar ou dedutivo) ou informações estatísticas extraídas de padrões. ${ }^{37}$ Esses padrões a serem classificados geralmente são grupos de medidas ou observações que definem pontos em um espaço multidimensional. Tais métodos são aplicados com diferentes finalidades: na análise exploratória de dados (métodos não-supervisionados), utiliza-se o conjunto de treinamento para criar novas categorias, ao invés de separar os dados de acordo com as categorias já existentes, e na classificação de amostras (métodos supervisionados), utiliza-se o conjunto de treinamento para classificar os dados de acordo com as categorias já existentes e nela organizá-los. ${ }^{38}$

Assim, o objetivo geral do trabalho desenvolvido foi avaliar fases sensoras de PVC na detecção de $17 \beta$-estradiol em meio aquoso utilizando a espectroscopia no infravermelho médio. Para tanto, estudos analíticos associados a análise quantitativa (limite de detecção, limite de quantificação, inclinação da curva analítica e coeficiente de determinação) e análise qualitativa (análise por componentes principais e modelo de classificação SIMCA) foram realizados.

\section{PARTE EXPERIMENTAL}

\section{Materiais e reagentes}

Foram utilizados os reagentes, em grau analítico, metanol (Dinâmica), tetrahidrofurano (THF) (Êxodo Científica); cloreto de sódio (Dinâmica). O PVC foi fornecido pela Tiletron ${ }^{\circledR}$, enquanto o ftalato de di-2-etil-hexil (DOP) pela Ciba-Geigy ${ }^{\circledR}$ e o $17 \beta$-estradiol (E2) pela farmácia de manipulação Finnofarma, da cidade de Serra Talhada - PE. A água para a preparação das soluções foi destilada e apresentou $\mathrm{pH}$ 7,0.

\section{Preparo das soluções}

A solução de referência do $17 \beta$-estradiol foi preparada em metanol, para uma concentração de $50 \mathrm{mg} \mathrm{L}^{-1}$. Os padrões sintéticos na faixa de concentração de 0,$0 ; 2,5 ; 5,0 ; 7,5 ; 10,0 ; 12,5$ e $15,0 \mathrm{mg} \mathrm{L}^{-1}$ foram preparados para a construção da curva analítica, com um tempo máximo de $1 \mathrm{~h}$ para uso no processo de extração e protegidos da luz, para evitar possível degradação. Esses padrões sintéticos foram diluídos em água destilada, sendo mantida uma percentagem final de metanol de $10 \%$ (v/v). Para a etapa de classificação das amostras (SIMCA), além das soluções indicadas acima, trabalhou-se com soluções de concentrações mais baixas, 0,$5 ; 1,0 ; 1,5$ e $2,0 \mathrm{mg} \mathrm{L}^{-1}$, totalizando 11 soluções de concentrações diferentes. As soluções do branco foram preparadas apenas com água destilada e 10\% (v/v) de metanol. Todos os experimentos foram realizados à temperatura ambiente média de $25 \pm 2{ }^{\circ} \mathrm{C}$.

\section{Preparação do filme de PVC}

Os filmes poliméricos foram obtidos pela técnica de evaporação de solvente (casting). ${ }^{15-16,39} \mathrm{O}$ PVC e o plastificante DOP foram 
dissolvidos em THF e após completa dissolução, o sistema polimérico foi transferido para uma placa de Petri (diâmetro de $10 \mathrm{~cm}$ ) e mantido por quatro dias até a secagem completa do filme, na temperatura ambiente, sem controle de temperatura nem umidade. A massa total para composição utilizada foi de $2,0000 \mathrm{~g}$ de PVC e $0,5 \mathrm{~mL}$ de DOP para 40,0 mL de THF.

\section{Extração do 17ß-estradiol e análise no infravermelho}

Um espectrofotômetro FTIR marca Perkin-Elmer ${ }^{\circledR}$, modelo Frontier foi utilizado para obtenção dos espectros na faixa de 600 a $4000 \mathrm{~cm}^{-1}$ (região do MID), usando o acessório universal de reflectância total atenuada (UATR). As condições de leitura foram resolução de $8 \mathrm{~cm}^{-1}, 8$ varreduras. Para as análises de extração do $17 \beta$-estradiol na fase sensora, inicialmente, os filmes de PVC (espessura de $0,17 \pm 0,02 \mathrm{~mm}$ ) foram cortados nas dimensões de $1,0 \mathrm{~cm} \times 1,3 \mathrm{~cm}$, codificados e marcados com um pequeno corte lateral direito, para garantir sempre o mesmo posicionamento nas análises (Figura 1a).

Antes do processo de extração, um espectro de infravermelho de cada fase foi adquirido para servir como referência (Figura 1b). Em seguida, a fase sensora foi introduzida em um frasco (com a solução do analito nas concentrações supracitadas, $1,5 \mathrm{~mol} \mathrm{~L}^{-1} \mathrm{de}$ $\mathrm{NaCl}$ e uma barra magnética) e mantido dentro do frasco, sob agitação constante padronizada, durante o tempo identificado no estudo cinético (Figura 1c). Após isso, a fase sensora polimérica foi retirada da solução, seca com papel absorvente e mais uma vez realizada a obtenção do espectro inserindo o filme polimérico na superfície do cristal do suporte UATR (Figura 1b). Todo esse procedimento é ilustrado na Figura 1.

\section{Figuras de mérito}

Foram construídas curvas analíticas em diversos comprimentos de onda e avaliadas para as extrações de soluções de E2, em triplicata: coeficiente de determinação $\left(\mathrm{R}^{2}\right)$; sensibilidade da calibração, avaliada com a inclinação da curva analítica (S); limite de detecção (LD) calculado a partir do desvio padrão do branco (Sb) e um fator de confiança 3 e o limite de quantificação (LQ). Para o cálculo do desvio padrão do branco ( $\mathrm{Sb}$ ), foram utilizados os dados referentes a cinco fases sensoras de PVC que entraram em contato com soluções do branco.

Todos os dados foram submetidos a pré-processamentos para corrigir efeitos indesejáveis como o ruído e o espalhamento da radiação. Dessa forma, foram selecionadas as regiões de trabalho e avaliados os dados sem tratamento e aplicadas às técnicas de normalização, correção de linha de base, suavização Savitzky-Golay (com janela de 7 pontos) e derivação ( $1^{\text {a }}$ derivada com filtro Savitzky-Golay, usando polinômio de $2^{\circ}$ grau e variando janelas de 7, 9 e 11 pontos), no intuito de identificar o melhor tratamento de dados, utilizando para isto o programa The Unscrambler X, versão 10.2 (CAMO S.A.).

\section{Aspectos cinéticos}

No estudo cinético foi investigado o tempo de extração para o $17 \beta$-estradiol em fases sensoras de PVC. Para tanto, foram construídas curvas de sinal analítico em relação ao tempo de exposição da fase sensora a uma solução 7,5 $\mathrm{mg} \mathrm{L}^{-1}$ de $17 \beta$-estradiol em metanol. Essa concentração foi escolhida com base na solubilidade do composto, pois conforme indicado na literatura o E2 apresenta uma solubilidade de $13 \mathrm{mg} \mathrm{L}^{-1} \mathrm{em}$ água, ${ }^{39}$ então, para o estudo cinético optou-se por trabalhar em uma concentração intermediária do composto. Os tempos investigados foram de 10, 15, 20, 30, 45, 60, 90 e 120 min, a cada análise uma nova fase sensora foi utilizada.

\section{Regeneração da fase sensora}

Para verificar se as fases sensoras poderiam ser reutilizadas após a interação com o analito, realizou-se uma investigação do número de ciclos que seria possível utilizar para mesma fase sensora sem perda de sinal analítico. O mesmo procedimento de extração do analito foi realizado e após cada ciclo de interação, a fase sensora era inserida em uma solução de lavagem, contendo água e metanol (1\% (v/v)). Para cada ciclo de limpeza era registrado um espectro e assim monitorou-se o sinal analítico no decorrer de 10, 20, 30, 40, 50 e $60 \mathrm{~min}$. O acompanhamento desses ciclos se deu até que o sinal de absorbância do analito diminuísse. Após isso, a fase sensora era submetida à outra solução de mesma concentração e assim sucessivamente, até 9 ciclos de utilização para cada fase sensora.

\section{Análise quimiométrica (PCA e SIMCA)}

Os dados adquiridos foram exportados e organizados em formato de matriz de dados espectrais. Nesse formato, as variáveis espectrais são representadas nas colunas, enquanto as amostras são representadas pelas linhas. Amostras anômalas (interpretadas aqui, como amostras que estão fora de um intervalo de confiança baseando-se nos valores de scores, obtidas via PCA, para um dado conjunto de amostras), foram identificadas inicialmente através da estatística entre os parâmetros $T^{2}$ Hotelling e $Q$ residual. Através do gráfico formado pelos valores obtidos de cada amostra para tais valores estatísticos, verificamos quão distante as amostras estão de um ponto central e, definindo limites, podemos selecionar quais amostras devem ser utilizadas na modelagem. Os valores $T^{2}$ Hotelling estão relacionados com os scores normalizados e acumulados obtidos, enquanto os valores $Q$ residual representa a soma dos quadrados para cada uma das amostras na matriz E (matriz residual de PCA). ${ }^{40,41}$ Dos 124 espectros analisados, foram identificadas somente 23 amostras outliers com a presença do $17 \beta$-estradiol. Assim, após a retirada delas, foram utilizadas 71 espectros para o grupo COM e 30 para o grupo SEM a presença do hormônio.

Posteriormente, o conjunto de dados foi submetido a análise de

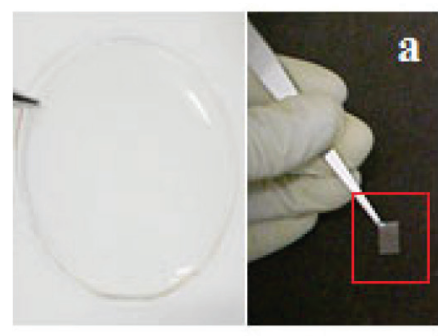

Filme de PVC (fase sensora)

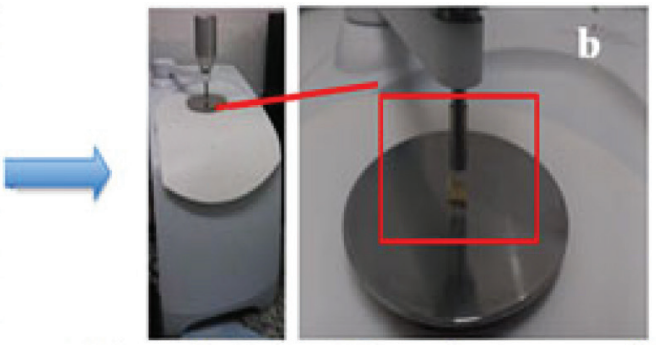

Sistema de inserç̧o da amostra para a medida

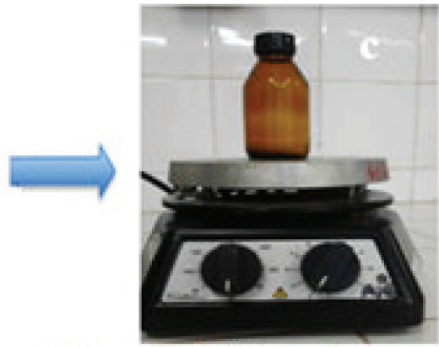

Extraçào de E2 com a fase sensora

Figura 1. Esquema do procedimento realizado para obtenção dos dados. (a)corte da fase sensora, (b)análise espectroscópica no UATR e (c)meio aquoso sob agitação onde a fase sensora era exposta por tempo deteminado 
componentes principais (PCA), um poderoso método de redução de dados amplamente utilizado em análises exploratórias. ${ }^{38}$

Para a classificação supervisionada, foi utilizado o modelo SIMCA, um método de classificação baseado exclusivamente na PCA das classes independentes analisadas. ${ }^{38,41-43}$ Ambos os modelos foram construídos no ambiente MATLAB R2014b versão 8.4 (MathWorks, Inc., Natick, USA) juntamente com o auxílio da interface PLSToolbox versão 7.9.3 (Eigenvector Research, Inc., Manson, USA) e rotinas construídas no laboratório.

Tanto para PCA quanto para a modelagem via SIMCA, os dados foram pré-processados com centragem na média. O número de componentes principais (PCs) para o modelo SIMCA foram escolhidos a partir do método de validação cruzada via leave-one-out, sendo 1 PCs com variância acumulada de $85,4 \%$ para o grupo sem a presença do analito e 4 PCs com variância acumulada de $96,1 \%$ para o grupo contendo analito.

\section{Validação estatística para o modelo SIMCA}

A validação estatística para o modelo SIMCA foi efetuada via: acurácia (AC), sensibilidade (SENS) e especificidade (SPEC). A acurácia está relacionada com a porcentagem de amostras que foram corretamente classificadas de acordo com suas respectivas classes. A sensibilidade mede a proporção de resultados positivos (neste caso, sem a presença de $17 \beta$-estradiol) corretamente identificados. Por outro lado, a especificidade mede a proporção de resultados negativos (neste caso, com a presença de $17 \beta$-estradiol) identificadas corretamente em seus respectivos grupos. ${ }^{41}$ Tais parâmetros podem ser calculados de acordo com as seguintes equações:

$$
\begin{gathered}
A C(\%)=\left(\frac{T P+T N}{T P+F P+T N+F N}\right) * 100 \\
\operatorname{SENS}(\%)=\left(\frac{T P}{T P+F N}\right) * 100
\end{gathered}
$$

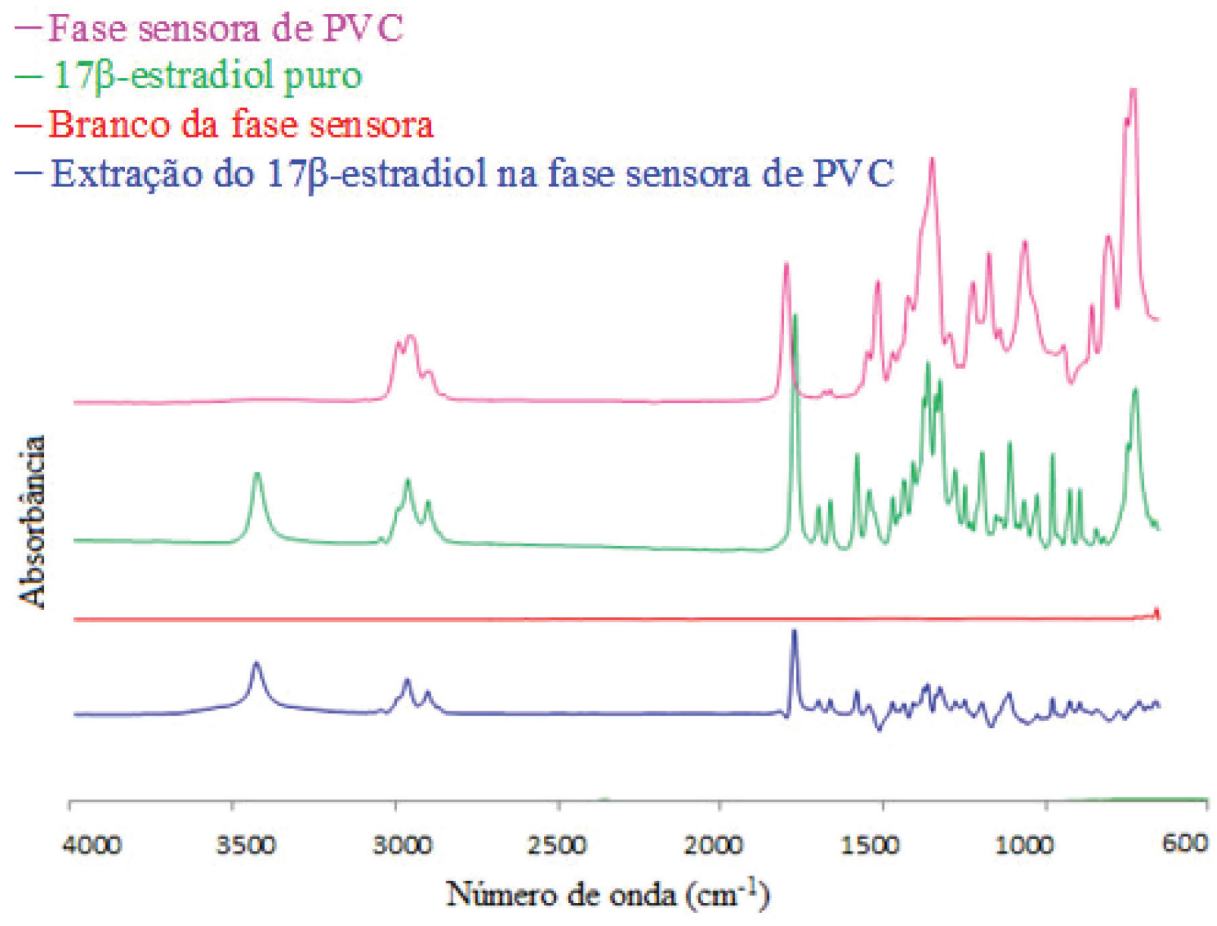

$$
\operatorname{SPEC}(\%)=\left(\frac{T N}{T N+F P}\right) * 100
$$

em que AC significa acurácia, SENS significa sensibilidade e SPEC significa especificidade. TP é definido como o número de casos verdadeiros positivos, $\mathrm{TN}$ como verdadeiros negativos, $\mathrm{FP}$ como falsos positivos e FN como falso negativo, sendo esses valores obtidos a partir dos resultados de classe para grupo de predição estimado pelo modelo SIMCA.

\section{RESULTADOS E DISCUSSÃO}

\section{Aplicação da fase sensora de PVC na detecção do $17 \beta$-estradiol em água}

Na Figura 2 é apresentado o espectro da fase sensora de PVC, do pó de $17 \beta$-estradiol puro, do branco da fase sensora de PVC e o espectro da fase sensora após extração de E2, durante 30 min, na concentração de $15 \mathrm{mg} \mathrm{L}^{-1}$.

Com base na impressão digital dos espectros do infravermelho médio, as bandas de absorção típicas para o PVC são identificadas próximas a $2970 \mathrm{~cm}^{-1}$ (estiramento $\mathrm{C}-\mathrm{H}$ do $\mathrm{CHCl}$ ); $2875 \mathrm{~cm}^{-1}$ (estiramento $\mathrm{C}$ - $\mathrm{H}$ do $\mathrm{CH}_{2}$ ); 1737, 1468, $1435 \mathrm{~cm}^{-1}$ (deformação $\mathrm{CH}_{2}$ ); banda entre 1399 a $1227 \mathrm{~cm}^{-1}$ (deformação $\mathrm{C}-\mathrm{H}$ do $\mathrm{CHCl}$ ); 1128 e $1076 \mathrm{~cm}^{-1}$ (estiramento $\mathrm{C}-\mathrm{C}$ ), $965 \mathrm{~cm}^{-1}$ (rotação $\mathrm{CH}_{2}$ ). ${ }^{15,42,44}$

As bandas correspondentes aos estiramentos vibracionais $\mathrm{C}-\mathrm{Cl}$ estão na região entre 715 a $667 \mathrm{~cm}^{-1}$. Essas bandas são de origens complexas e dependem da estrutura conformacional do polímero e da posição espacial dos átomos próximos às ligações $\mathrm{C}$ - $\mathrm{Cl}$. As outras bandas do espectro correspondem às diferentes vibrações C-C e $C-H .^{15,44}$

A literatura reporta que a associação de plastificante (aditivos de baixo peso molecular) ao PVC pode resultar em uma matriz polimérica flexível com características químicas e físicas desejáveis, 
o que favorece também o aumento do volume livre entre as cadeias poliméricas, podendo assim facilitar a penetração do analito na matriz e aumentar a interação da fase sensora com o contaminante ambiental de interesse. ${ }^{42}$ As bandas correspondentes ao plastificante ftalato de di-2-etil-hexil (DOP) utilizado na confecção dos filmes nesse trabalho são bastante complexas e se superpõem às bandas do próprio PVC. As principais bandas que caracterizam o DOP estão nas regiões compreendidas entre 1798 a $1646 \mathrm{~cm}^{-1} ; 1316$ a $1264 \mathrm{~cm}^{-1}$ e 1167 a $1103 \mathrm{~cm}^{-1} .^{15}$

O espectro de infravermelho do pó do $17 \beta$-estradiol possui as seguintes regiões de absorção e maior interesse espectral, em $3420 \mathrm{~cm}^{-1}$ atribuída ao estiramento de $O H$, em ligação de hidrogênio intermolecular. As regiões de 3014 a $2850 \mathrm{~cm}^{-1}$ características da deformação $C$ - $H$ aromático $\left(2964 ; 2923 ; 2872 ; 2851 \mathrm{~cm}^{-1}\right)$ da ligação de $\mathrm{CH}_{3}$ e $\mathrm{CH}_{2}$ simétricos e assimétricos. Em 1696 a $1454 \mathrm{~cm}^{-1}$ a região é correspondente à deformação axial de $C$ - $C$ do anel e nas regiões de 1227 a $1270 \mathrm{~cm}^{-1}$ atribuída à deformação angular $O H$. Ainda, as bandas na região de $1114 \mathrm{a} 1062 \mathrm{~cm}^{-1}$ atribuídas à deformação axial de $C$ - $O$ e as regiões de 923 a 810 e 805 a $771 \mathrm{~cm}^{-1}$ à deformação angular fora do plano de $\mathrm{CH}_{2}{ }^{44,45}$

A partir da Figura 2 pode ser observada uma vantagem para utilização dos filmes de PVC como fase sensora, pois não existem bandas na principal região de detecção do analito de interesse (região de 3420 e $1696 \mathrm{~cm}^{-1}$ ) e, sendo assim, não irão ocorrer interferências espectrais referentes às informações da fase sensora em contraste com os dados espectrais do analito nesses intervalos de comprimentos de onda.

\section{Estudo cinético}

Trabalhando-se com o tempo de equilíbrio pode-se obter melhores resultados, pois a sensibilidade do método é aumentada. ${ }^{21,46}$ Neste estudo utilizamos o número de onda em $1696 \mathrm{~cm}^{-1}$, por ser uma das principais regiões de detecção do E2. Na Figura 3 é possível observar que houve a saturação da fase sensora após 30 min de extração, sendo então esse o tempo escolhido para agitação dos filmes nas soluções de E2.

Vale ressaltar que foi realizada a investigação apenas para a concentração de 7,5 $\mathrm{mg} \mathrm{L}^{-1}$. Para concentrações menores possivelmente o tempo para equilíbrio da fase sensora seria maior e pela proposta era necessário saber um comportamento médio do sistema, por isso escolheu-se uma concentração intermediária.

\section{Figuras de mérito}

As figuras de mérito avaliadas neste estudo foram: coeficiente de determinação $\left(\mathrm{R}^{2}\right)$, o desvio padrão do branco $\left(\mathrm{SD}_{\text {branco }}\right)$, a sensibilidade da calibração (S) (dada pela inclinação da curva analítica), o limite de detecção (LD) e o limite de quantificação (LQ), calculadas para diferentes números de onda $\left(\mathrm{cm}^{-1}\right)$. Para a curva analítica, os melhores resultados foram obtidos aplicando o tratamento suavização Savitzky-Golay (com janela de 7 pontos), para os comprimentos de onda $3420 \mathrm{~cm}^{-1}, 1696 \mathrm{~cm}^{-1}, 1498 \mathrm{~cm}^{-1}, 1098 \mathrm{~cm}^{-1}$ e $874 \mathrm{~cm}^{-1}$.

Na Tabela 1, observam-se os principais resultados obtidos para os números de onda destacados.

Os limites de detecção obtidos pelo método proposto são satisfatórios e apresentam-se como promissores quando observado trabalhos semelhantes, em que também foram utilizadas fases sensoras poliméricas para detectar contaminantes orgânicos em água. Silva obteve em seu estudo de determinação de compostos BTEX em água por meio de fases sensoras de PVC um limite de detecção de 0,40 a $2,4 \mathrm{mg} \mathrm{L}^{-1}$. Souza, em seu estudo com fases sensoras de poliuretano na determinação de tolueno em água, encontrou $\mathrm{LD}$ variando de 1,4 a $6,8 \mathrm{mg} \mathrm{L}^{-1}$, esses dados com medidas de transmitância.

Em se tratando de contaminantes emergentes, compreende-se que esses compostos por não serem legislados, não há na literatura limites de detecção, assim, sinalizando a importância de estudos

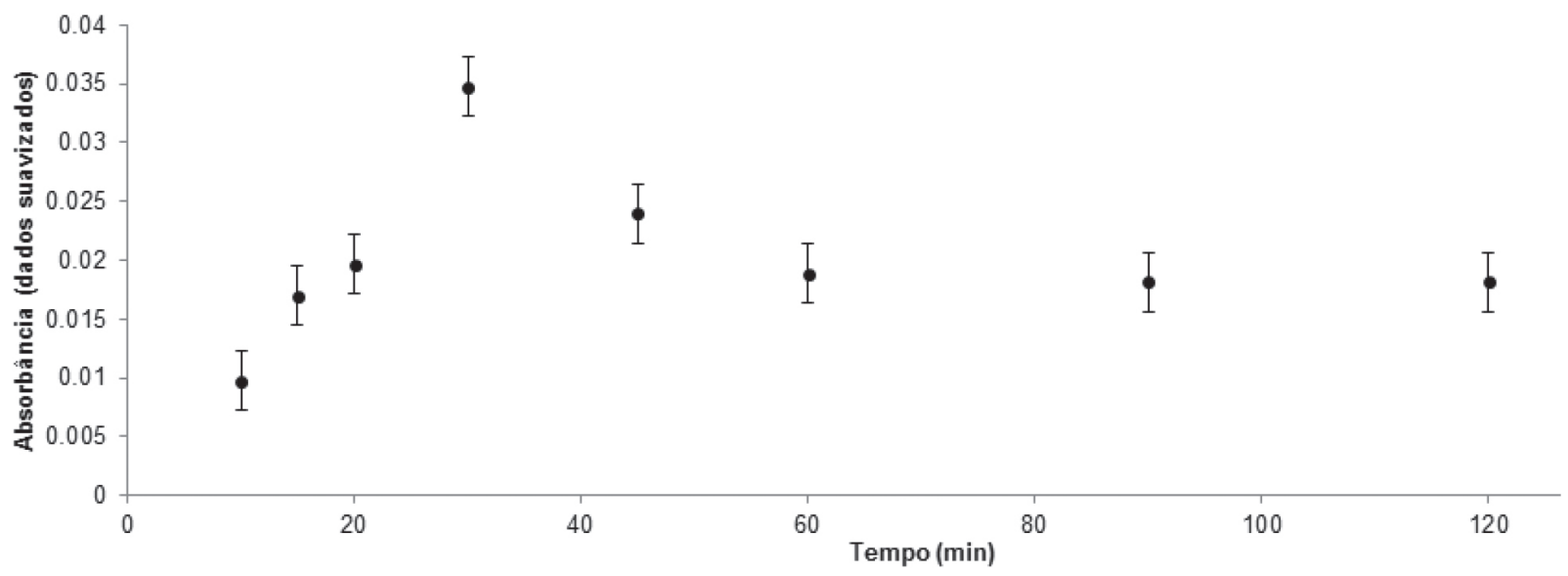

Figura 3. Perfil do tempo de extração de fases sensoras de PVC em contato com soluções de 7,5 $\mathrm{mg} \mathrm{L}^{-1}$ de E2. As barras representam o desvio padrão médio. Dados suavidados com filtro Savitzky-Golay, janela de 7 pontos, número de onda em $1696 \mathrm{~cm}^{-1}$

Tabela 1. Figuras de mérito obtidas para o E2 extraído em PVC por $30 \mathrm{~min}$, em diferentes números de onda $\left(\mathrm{cm}^{-1}\right)$. Dados médio, suavizados com filtro Savitzky-Golay, janela de 7 pontos

\begin{tabular}{|c|c|c|c|c|c|c|}
\hline $\begin{array}{l}\text { Número de onda } \\
\left(\mathrm{cm}^{-1}\right)\end{array}$ & Banda característica & $\mathrm{R}^{2}$ & $\begin{array}{l}\mathrm{SD}_{\text {branco }} \\
\left(\mathrm{mg} \mathrm{L}^{-1}\right)\end{array}$ & $\begin{array}{c}\mathrm{S} \\
\left(\mathrm{L} \mathrm{mg}^{-1}\right)\end{array}$ & $\begin{array}{c}\mathrm{LD} \\
\left(\mathrm{mg} \mathrm{L}^{-1}\right)\end{array}$ & $\begin{array}{c}\mathrm{LQ} \\
\left(\mathrm{mg} \mathrm{L}^{-1}\right)\end{array}$ \\
\hline 3420 & Estiramento de $\mathrm{OH}$, em ligação de hidrogênio intermolecular & 0,98 & $2,4 \times 10^{-3}$ & $2,4 \times 10^{-3}$ & 3,1 & $1,0 \times 10^{-1}$ \\
\hline 1696 & Deformação axial de C-C do anel & 0,99 & $3,0 \times 10^{-4}$ & $1,2 \times 10^{-2}$ & $8,9 \times 10^{-1}$ & 2,9 \\
\hline 1498 & Deformação axial de C-C do anel & 0,94 & $1,1 \times 10^{-3}$ & $1,2 \times 10^{-2}$ & 2,9 & 9,6 \\
\hline 1098 & Deformação axial de C-O & 0,91 & $4,0 \times 10^{-4}$ & $5,0 \times 10^{-4}$ & 2,5 & 8,4 \\
\hline 874 & Deformação angular fora do plano de $\mathrm{CH}_{2}$ & 0,90 & $4,0 \times 10^{-4}$ & $5,0 \times 10^{-4}$ & 2,8 & 9,5 \\
\hline
\end{tabular}


fundamentais que apontem os níveis de concentração no ambiente e exijam, na análise preliminar, um custo baixo associado. Compreendese, também, que a técnica de sensores atrelada à espectroscopia IR não se ocupa do mérito de comparação a técnicas cromatográficas. No entanto, Singh e colaboradores reportam um ponto importante no que tange a espectroscopia de infravermelho na detecção de contaminantes emergentes, em especial ao alvo deste trabalho, $17 \beta$-estradiol. Os autores sinalizam que os dados gerados obtidos pela técnica espectroscópica, seja na região MIR ou NIR, podem ser usados como parâmetros de entrada em modelos de destino para avaliações de risco e programas de tomada de decisão para segurança ambiental de água e solos. ${ }^{47}$

\section{Avaliação da reutilização da fase sensora}

Diferentes fatores podem influenciar a entrada e saída de um analito em uma fase sensora, como o tamanho da molécula, os espaços livres na fase sensora, a superfície de interação do material polimérico e a temperatura. ${ }^{15,19-22}$. Para as fases sensoras de PVC utilizadas neste trabalho, as interações com o E2 mostraram-se reversíveis, indicando a possibilidade de reutilização da fase sensora. Observa-se na Figura 4 que não há perda de sinal analítico nos primeiros quatro ciclos, pois é possível perceber que mesmo se tratando de uma fase sensora já utilizada, a absorbância do analito em exposição durante 30 min à concentração inicial $\left(7,5 \mathrm{mg} \mathrm{L}^{-1}\right)$ permaneceu a mesma $(0,025)$, indicando que a fase pode ser reutilizada para outras análises. Ressalta-se aqui, que para cada ciclo esperou-se um tempo de $24 \mathrm{~h}$ para reutilização.

Dos nove ciclos analisados, observou-se que a partir do $5^{\circ}$ ao $9^{\circ}$ ciclos o sinal da absorbância diminui com um percentual de perda (\%) de 16, 20, 28, 32 e 36, respectivamente, tendo como $100 \%$ o valor inicial de absorbância 0,025 , o que configura, dessa forma, uma confiabilidade de 4 reutilizações da fase sensora de PVC, indicando que quanto mais usar, maior será a perda de sinal a partir do $5^{\circ}$ ciclo.

No decorrer da limpeza da fase sensora com água e metanol, em cada ciclo, observou-se um decaimento do sinal, indicando que o analito está saindo da fase sensora. A partir de 40 min a absorbância diminui significativamente até chegar em 60 min, onde a absorbância tende a zero (Figura 4). Assim, é indicado que a regeneração é eficiente e que a fase sensora poderá ser reutilizada. Também é importante ressaltar, que durante todas as análises realizadas, não se observaram mudanças nas bandas principais do filme de PVC, indicando que a composição inicial do material permanecia a mesma.

\section{Análise quimiométrica PCA e SIMCA}

Antes da utilização do modelo SIMCA, os dados foram inicialmente divididos em 2 grupos, sendo estes os grupos de treinamento e predição. Tal seleção de amostras foi realizada via algoritmo de Kennard-Stone (KS). ${ }^{48}$ Cerca de $70 \%$ ( $n=50$ para grupo COM e $n=21$ para grupo SEM) das amostras de cada grupo foram utilizadas para o grupo de treinamento (utilizado para construção do modelo SIMCA), enquanto $30 \%$ ( $n=21$ para grupo COM e $n=9$ para grupo SEM) foram utilizadas para o grupo de predição (utilizado para o cálculo das figuras de mérito de classificação como acurácia, sensibilidade e especificidade). A Figura 5 demonstra a análise de componentes principais para o conjunto de dados analisados.

Podemos perceber, principalmente pela Figura 5 (b), um princípio de formação de grupos referentes aos espectros obtidos a partir da presença e ausência do analito em questão. Tal característica corrobora com a vísivel diferenciação espectral em determinados comprimentos de onda, o que demonstra a possível viabilidade da análise exploratória juntamente com a espectroscopia UATR/FTIR na diferenciação de amostras.

O modelo SIMCA foi desenvolvido empregando os espectros IR das fases sensoras para o grupo sem e com a presença do analito com o objetivo de obter um modelo de classificação. Para as amostras do grupo sem a presença do analito $(n=21$ amostras para calibração e $\mathrm{n}=9$ amostras para previsão) foi utilizado 1 componente principal (85,4\% de variância acumulada) e para o grupo contendo o analito ( $\mathrm{n}=50$ amostras para calibração e $\mathrm{n}=21$ amostras para previsão) foram utilizadas 4 componentes principais (96,1\% da variância acumulada). No grupo de previsão, o modelo SIMCA, analisando 9 amostras referentes ao grupo sem a presença do analito e 21 amostras com a presença do analito, no nível de confiança de $95 \%$, alcançou uma acurácia de $80 \%$, com sensibilidade máxima de $100 \%$ e especificidade de $71,4 \%$. Portanto, o modelo SIMCA com elevada acurácia e sensibilidade para identificação de amostras sem a presença de $17 \beta$-estradiol em água é satisfatório e viável tecnicamente de ser implantado como um método de triagem.

\section{CONCLUSÕES}

Propusemos neste trabalho um estudo sobre o desempenho do poli(cloreto de vinila) - PVC como fase sensora para detecção de $17 \beta$-estradiol em água, por meio da espectroscopia no infravermelho

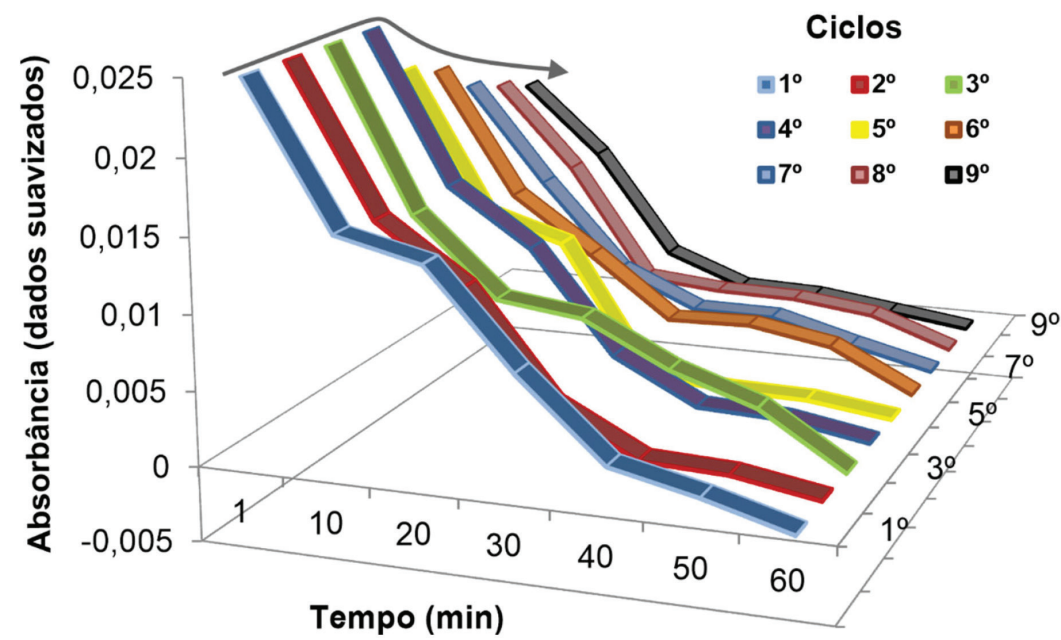

Figura 4. Gráfico em $3 D$ da reutilização da fase sensora em nove ciclos de extração, após agitação durante 30 min com uma solução de 7,5 mg $L^{-1}$ de E2 e acompanhamento de limpeza em solução de água/metanol (1\% v/v) por 60 min. Dados suavizados com filtro Savitzky-Golay, janela de 7 pontos, número de onda em $1696 \mathrm{~cm}^{-1}$ 

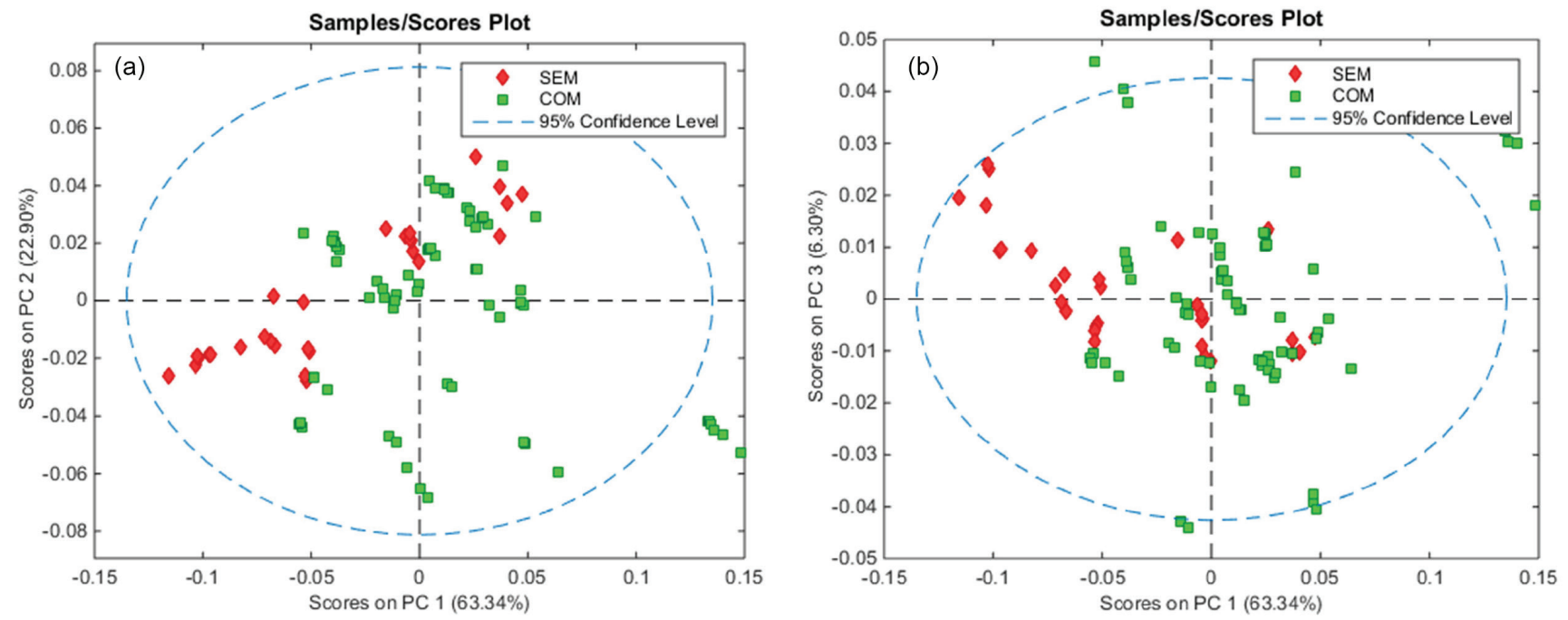

Figura 5. (a) Gráfico de scores utilizando PC1 x PC2 através de PCA; (b) Gráfico de scores utilizando PC1 x PC3 através de PCA

médio e quimiometria. Os resultados apontaram ótimos valores analíticos para a método avaliado, apresentando limites de detecção de $8,9 \times 10^{-1}$ a $2,9 \mathrm{mg} \mathrm{L}^{-1}$, limite de quantificação entre 2,9 a $1,0 \times 10 \mathrm{mg} \mathrm{L}^{-1}$, inclinação e coeficiente de determinação das curvas analíticas 5,0x10-4 a $1,2 \times 10^{-3} \mathrm{~L} \mathrm{mg}^{-1}$ e 0,90 a 0,99 , respectivamente, com o tratamento de suavização Savitzky-Golay (com janela de 7 pontos). A partir do estudo cinético realizado, observou-se, para a fase sensora de PVC em solução contaminada com o analito, um tempo de extração em torno de $30 \mathrm{~min}$. No estudo de reutilização da fase sensora, observou-se que as fases de PVC podem ser reutilizadas até 4 vezes. $\mathrm{Na}$ avaliação qualitativa por meio da análise das componentes principais (PCA), foi observada uma tendência na separação entre as amostras aquosas contaminadas e não contaminadas. Em relação ao SIMCA, conseguimos obter considerável taxa de acurácia e sensibilidade para identificação de amostras sem a presença de 17ß-estradiol em água, o que demonstra uma possível viabilidade da técnica espectroscópica em conjunto com análise multivariada para um futuro método de triagem rápido, barato, quimicamente verde e sofisticado.

\section{AGRADECIMENTOS}

Os autores agradecem à FinnoFarma (Serra Talhada-Pernambuco) pela doação $17 \beta$-estradiol, a Tiletron e Ciba-Geigy pelo PVC e DOP doadas para professora Glória Maria Vinhas (DEQ/UFPE), que nos forneceu para o desenvolvimento da pesquisa, ao CNPq (Bolsa PIBIC/ UFRPE), à FACEPE (PRONEX/NUQAAPE (APQ-0346-1.06/14)), ao Grupo de Instrumentação e Análise Química (Unidade Acadêmica de Serra Talhada / UFRPE) e à professora Maria Fernanda Pimentel Avelar (DEQ/UFPE) pelo uso dos softwares The Unscrambler X, versão 10.2 (CAMO S.A.) e MATLAB R2014b 8.4. D. L. D. Freitas gostaria de agradecer a CAPES/PPGQ/UFRN por todo o apoio financeiro. K. M. G. Lima agradece ao CNPq (Processo 303733/2017-9).

\section{REFERÊNCIAS}

1. Grover, D. P.; Zhou, J. L.; Frickers, P. E.; Readman, J. W. J.; J. Hazard. Mater. 2011, 185, 1005.

2. Queiroz, F. B.; Brandt, E. M.; Aquino, S. F.; Chernicharo, C. A.; Afonso, R. J.; Water Sci. Technol. 2012, 66, 2562.

3. Adeel, M.; Song, X.; Wang, Y.; Francis, D.; Yang, Y.; Environ. Int. 2016, 99, 107.

4. Reh, R.; Lincha, T.; Geyer, T.; Nodler, K.; Sauter, M.; Sci. Total Environ. 2013, $443,438$.
5. Lima, D. R. S.; Tonucci, M. C.; Libanio, M.; Aquino, S. F.; Eng. Sanit. Ambient. 2017, 22, 1043.

6. Montagner, C. C.; Vidal, C.; Acayaba, R. D.; Quim. Nova 2017, 40, 1094.

7. Wang, A.; Ding, Y.; Li, L. Dingding, D.; Mei, Q.; Zhuang, Q.; Cui, S.; He, X.; Talanta 2019, 192, 478.

8. Das, A; Sangaranarayanan, M. V.; Sens. Actuators, B 2018, 256, 775 .

9. Fernandes, A. N.; Giovanela, M.; Almeida, C. A. P.; Esteves, V. I.; Sierra, M. M. D.; Grassi, M. T.; Quim. Nova 2011, 34, 1526.

10. Schug, T. T.; Janesick, A.; Blumberg, B.; Heindel, J. J.; J. Steroid Biochem. Mol. Biol. 2011, 127, 3.

11. Freitas, E. V. C.; Barreto, F. M. S.; Neto, M. F. A.; Cavalcante, R. M. Águas Subterrâneas 2016, 30, 289.

12. Su, R.; Ruan, G.; Nie, H.; Xie, T.; Zheng, Y.; Du, F. Li, J.; J. Chromatogr. A. 2015, 1405, 23.

13. Linan, L. Z.; Bonon, A.; Lima, N. M. N.; Filho, R.; Manenti, F.; Chem. Eng. Trans. 2013, 32, 1699.

14. Raymundo-Pereira, P. A.; Campos, A. M.; Vicentini, F. C.; Janegitz, B. C.; Mendonça, C. D.; Furini, L. N.; Boas, N. V.; Calegaro, M. L.; Constantino, C. J. L. ; Machado, S. A. S.; Oliveira Jr, O. N.; Talanta 2017, 174, 652 .

15. Silva, A. M. S.; Tese de doutorado, Universidade Federal de Pernambuco, Brasil, 2008.

16. Silva, A. M. S.; Pimentel, M. F.; Raimundo Jr, I. M.; Almeida, Y. M. B.; Sens. Actuators, B 2009, 139, 222.

17. Lima, K. M. G.; Tese de doutorado, Universidade Estadual de Campinas, Brasil, 2009.

18. Hass, J.; Mizaikoff, B.; Annu. Rev. Anal. Chem. 2016, 9, 45.

19. Souza, J. D. C.; Júnior, R. V. A.; Vinhas, G. M.; Lima, N. B. D.; Brito, A. M. S. S.; Quim. Nova 2018, 41, 394.

20. Flavin, K.; Hughes, H.; Dobbyn, V.; Kirwan, P.; Murphy, K.; Steiner, H.; Mizaikoff, B.; Mcloughlin, P.P.; Intern. J. Environ. Anal. Chem. 2006, $86,401$.

21. Lima, M. G. K.; Raimundo Jr, I. M.; Pimentel, M. F.; Sens. Actuators, B 2007, 125, 229.

22. Yang, J.; Ramesh, A.; Analyst 2005, 130, 397

23. Lu, R.; Sheng, G.; Li, W.; Yu, H.; Raichlin, Y.; Katzir, A.; Mizaikoff, B.; Angew Chem., Int. Ed. 2013, 52, 2265.

24. Pejcic, B.;Leign, B.; Myres, M.; Ross, A.; Raichiln, Y.; Katzir, A.; Lu, R.; Mizaikoff, B.; Org. Geochem. 2013, 55, 63.

25. Stach, R.; Pejcic, B.; Crooke, E.; Myres, M.; Mizaikoff, B.; Anal. Chim. Acta. 2015, 87, 12306. 
26. Liu, S.; Hu, Q.; Zheng, J.; Xie, L.; Wei, S.; Jiang, R.; Zhu, F.; Liu, Y.; Ouvang, G.; J. Chromatogr. A 2016, 1450, 9.

27. Lu, R.; Li, W. W.; Mizaikoff, B.; Katzir, A.; Raichlin, Y.; Sheng, G. P.; Yu, H. Q.; Nat. Protoc. 2016, 11, 377.

28. Regan, F.; Meaney, M.; Vos, J. G.; MacCraith, B. D.; Walsh, J. E.; Anal. Chim. Acta 1996, 334, 85.

29. Mizaikoff, B.; Water Sci. Technol. 2003, 47, 35.

30. Silva, A. M. S.; Viana, E. A.; Pimentel, M. F.; Almeida, Y. M. B.; Raimundo Jr, I. M.; J. Braz. Chem. Soc. 2011, 22, 1470.

31. Mizaikoff, B.; Proc. SPIE Int. Soc. Opt. Eng. 1999, 3849, 7.

32. White, C.; Pejcic, B.; Myres, M.; Qi, X.; Sens. Actuators, B 2014, 193, 70 .

33. Yang, J. Tsai, S. S.; Anal. Chim. Acta. 2002, 462, 235.

34. Howley, R.; MacCraith, B. D. Dwyer, K. O.; Kirwan, P.; McLoughlin, P.; Vib. Spectrosc. 2003, 31, 271.

35. Jones, Y. K.; Jonhson, M. M.; Li, Z.; Josse, F.; Hossenlopp, J.; M. IEEE Sens. J. 2005, 5, 1175.

36. Mccue, R. P.;Walsh, J. E.; Walsh, F. Regan, F.; Sens. Actuators, B 2006, 114,438 .

37. Moita Neto, J. M.; Moita, G. C.; Quim. Nova 1998, 21, 467.
38. Beebe, K. R.; Randy, J. P.; Seasholtz, M. B.; Chemometrics: A practical guide, $1^{\mathrm{a}}$ ed., Wiley Interscience: New York, 1998.

39. Jones, D.; Medlicott, N.; J. Int. J. Pharm. 1995, 114, 257.

40. Paraskevaidi, M.; Morais, C.; Freitas, D. L. D.; Gomes de Lima, K. M.; Mann, D. M. A.; Allsop, D.; Martin-Hirsch, P. L.; Martin, F. L.; Analyst 2018, 143, 5959.

41. Ramus, B.; Smilde, A. K.; Anal. Methods 2014, 6, 2812.

42. Beltrán, M.; Marcilla, A.; Garcia, J. C.; Eur. Polym. J. 1997, 33, 1271.

43. Oliveira, G. H. F.; Murray, S. C.; Cunha Júnior, L. C.; Lima, K. M. G.; Morais, C. L. M.; Teixeira, G. H. A.; Môro, G. V.; J. Cereal Sci. 2020, $91,102861$.

44. Silverstein, R. M.; Webster, F. X.; Kiemle, D. J.; Identificação espectrométrica de compostos orgânicos, $7^{\text {a }}$ ed., LTC: Rio de Janeiro, 2013

45. Minaev, B. F.; Minaeva, V. A.; Biopolym. Cell. 2006, 22, 363.

46. Souza, J. C.; Dissertação de mestrado, Universidade Federal de Pernambuco, Brasil, 2017.

47. Singh, B.; Malley, D. F.; Farenhost, A.; Willams, P.; Biosens. Bioelectron. 2012, 126, 15.

48. Kennard, R. W.; Stone L. A.; Technometrics 1996, 11, 137. 